\title{
Reliability of inertial sensors in the assessment of patients with vestibular disorders: a feasibility study
}

\author{
Sathish K. Sankarpandi 1,2,5* Alice J. Baldwin ${ }^{3}$, Jaydip Ray ${ }^{3,2}$ and Claudia Mazzà ${ }^{1,4}$
}

\begin{abstract}
Background: Vestibular disorders affect an individual's stability, balance, and gait and predispose them to falls. Traditional laboratory-based semi-objective vestibular assessments are intrusive and cumbersome provide little information about their functional ability. Commercially available wearable inertial sensors allow us to make this real life assessments objective, with a detailed view of their functional abilities. Timed Up and Go (TUG) and Postural Sway tests are commonly used tests for gait and balance assessments. Our aim was to assess the feasibility, testretest reliability and ability to classify fall status in individuals with vestibular disorders using parameters derived from the commercially available wearable system (inertial sensors and the Mobility Lab Software, APDM, Inc.).

Methods: We recruited 27 individuals diagnosed either with unilateral or bilateral vestibular loss on vestibular function testing. Instrumented Timed Up and Go (iTUG) and Postural Sway (iSway) were administered three times during the first session and then repeated at a similar time the following week. To evaluate within and between sessions reliability of the parameters the Intra-Class Correlation coefficient (ICC) was used. Subsequently, the ability of reliable parameters (ICC $\geq 0.8$ ) to classify fallers from non-fallers was estimated.
\end{abstract}

Results: The iTUG test parameters showed good within and between sessions' reliability with mean ICC (between-sessions) values of $0.81 \pm 0.17$ and $0.69 \pm 0.15$, respectively. For the iSway test, the relative figures were; $0.76 \pm 0.13$ and $0.71 \pm 0.14$, respectively. A retrospective falls classification analysis with past 12 months falls history data yielded an accuracy of $66.70 \%$ with an area under the curve of 0.79 . Mean Distance from centre of COP $(\mathrm{mm})$ of accelerometer's trajectory $\left(\mathrm{m} / \mathrm{s}^{2}\right)$ from the iSway test was the only significant parameter to classify fallers from non-fallers.

Conclusions: Using a commercially available wearable system a subset of reliable iTUG and iSway parameters were identified and their ability to classify fallers were estimated. These parameters have potential to augment assessments of vestibular patients to enable clinicians and therapists to provide objective, tailored, personalised interventions for their gait and postural control and also to objectively evaluate and monitor the efficiency of their interventions.

Keywords: Vestibular disorders, Reliability, OPAL inertial sensors, TUG and sway tests

\footnotetext{
* Correspondence: sathishkumarsankar@gmail.com

${ }^{1}$ INSIGNEO Institute for in silico Medicine, University of Sheffield, Sheffield, UK

${ }^{2}$ School of Health Sciences, University of Salford, Manchester, UK

Full list of author information is available at the end of the article
} 


\section{Background}

The ability of an individual to maintain posture and balance in both static and dynamic modes is essential for safe, independent living. Vestibular pathology is a common phenomenon frequently seen in the ageing population. Individuals with vestibular pathology commonly present with unsteadiness and imbalance, both of which can lead to falls and fragility fractures [1]. Falls, especially in the elderly, are associated with increased morbidity and can have a negative impact on socialisation resulting in a loss of independence and consequent reduced quality of life $[2,3]$. A recent study found that $80 \%$ of fallers admitted to Emergency departments in the UK had symptoms of an underlying vestibular impairment [4]. In 2010, the UK spent $£ 203$ million treating individuals who had sustained falls highlighting a substantial financial expenditure and significant pressure on the health care services [5]. A prompt, accurate diagnosis and management of the instability of patients with vestibular deficits would reduce the number of fallers and associated morbidity and healthcare expenditure.

Clinical tests of vestibular patients include the Romberg's test, Tandem Gait, and Fukuda's stepping tests which tend to be inaccurate and not always reproducible. The Timed up and Go (TUG) test used widely by other specialities is more objective and repeatable and could be used to augment the functional assessment of the vestibular patients.

Vestibular assessments generally available in dedicated balance clinics are laboratory based and include rotation tests, electronystagmography (ENG), videonystagmography (VNG) and occasionally Computerised Dynamic Posturography (CDP) [6]. Most provide an estimate of the function of the vestibular organ whilst only the CDP has the advantage of being able to quantify static balance functions related to posture and stability. However, it is cumbersome and intrusive, as it usually requires the patient to be restrained in a harness whilst the floor is moved and does not reflect the patient's natural setting [7]. Its cost is not negligible and its availability is limited to only a few tertiary centres. Following these tests most patients will receive vestibular rehabilitation, which may be delayed depending on local availability of resources. Therefore, the need for a simple, portable, quick, objective, reliable and repeatable assessment technique that would allow clinicians to diagnose deficits in real life situations, monitor the rehabilitation progress and predict falls is imperative.

The Timed up and Go (TUG) and postural sway tests used widely by other specialities and could be used to augment the functional assessment of the vestibular patients. Nevertheless, results these tests suffer from subjective assessments. Currently, instrumented version of TUG and Sway tests using inertial sensors show that results of these tests are objective, reproducible and better than traditional assessments [8-12]. Inertial wearable sensors (triaxial gyroscopes and accelerometers) are small, lightweight and inexpensive devices are being increasingly explored to test balance impairments in individuals with neurodegenerative conditions and vestibular impairments $[10,13]$. Although research shows that inertial sensors are capable to screen participants with vestibular disorders it is not widely used in the everyday clinical environment. This may be because i) the inertial sensor systems are not yet fully translated for a simple use by a clinician ii) feasibility and reliability of the system in the clinical environment is not well reported.

Recently, commercial exploration of inertial sensors system led to the development of systems that are portable, automated, easy to use, and setup. This preliminary study aims to investigate the feasibility of use and reliability of the one of the commercially available inertial sensor systems known as Mobility Lab ${ }^{\mathrm{Tm}}$ (APDM, Inc.) [14]. In particular, it aims to assess the test-retest reliability of parameters extracted from instrumented Timed Up and Go (TUG) and Sway tests and also to investigate the ability of reliable parameters to classify fallers in the cohort of participants with vestibular impairments.

\section{Methods \\ Participants}

Twenty-seven patients ( 7 males and 20 females, age range: 40-81 years) were recruited from those that visited the Neurotology Clinics. An informed consent was obtained prior to participation.

Inclusion criteria were:

i) Unilateral labyrinthine weakness and

ii) Bilateral labyrinthine loss, as indicated by their vestibular function tests.

All underwent objective vestibular assessments in the vestibular laboratory using Videonystagmography, Electronystagmography, Caloric testing, and Posturography to measure labyrinthine weakness / failure.

Exclusion criteria were:

i) Active Meniere's disease and vestibular hydrops,

ii) Benign Paroxysmal Positional Vertigo (BPPV)

iii) History of dizziness related to Transient Ischaemic Attacks (TIAs) due to the fluctuant nature of symptoms.

iv) Individuals with central pathologies including epilepsy, significant visual impairment, excessive alcohol consumption and those on vestibular sedatives were also excluded. 
To recruit a homogenous vestibular cohort of participants and exclude every other non-vestibular cause of balance problems, a detailed medical history was undertaken and a full list of concurrent medication was cross-checked for any contribution towards vestibular symptoms and / or falls.

The National Research Ethics committee West Midlands-Edgbaston, reference number 14/WM/0146, approved the study.

\section{Protocol}

The Mobility Lab ${ }^{\text {Tw }}$ system was used to record, process and store data obtained from previously validated algorithms $[10,12,14]$. The system is composed of six different Magnetic Inertial Measurement Units (MIMUs), each of which contains 3 -axis accelerometers $( \pm 6 \mathrm{~g}), 3$-axis gyroscopes $\left( \pm 2000^{\circ} / \mathrm{s}\right.$ for the yaw and roll axes, and $\pm 1500^{\circ} / \mathrm{s}$, for the pitch axis) and 3-axis magnetometers ( \pm 6 Gauss). MIMU's were attached around wrists, sternum, chest and shanks of the participants using adjustable Velcro straps. All the sensors were configured for synchronised recording and real-time data acquisition at a sampling rate of $128 \mathrm{~Hz}$.

The participants were asked to perform an iTUG and an iSway test following the protocols suggested by the system manufacturer [14]. In order to assess within and between sessions reliability, each patient underwent two sessions, one week apart from each other, and three repetitions of both iTUG and iSway tests were performed in each session.

All patients and operators were asked to fill in a questionnaire on how easy or difficult it was to set up and use the sensors (1: Easy, 2: Slight difficulty, 3: Significant difficulty, 4: Not possible to use). Operators were additionally asked to record the time taken from setup to obtaining the results.

\section{ITUG}

The TUG is a test widely used the test to assess balance and mobility $[15,16]$. In its instrumented version, participants were instructed to rise from a standard armless chair, walk in a straight line for seven metres, turn $180^{\circ}$, return to the chair and sit down. Individuals were requested not to elevate themselves from the chair with their hands when sitting down and standing up and to walk at their normal ambient pace. The tape was used to mark the floor at the seven metres and these marks were clearly shown to the participants. Thirtytwo parameters were obtained from the sensor signals. The parameters extracted are grouped based on individual subcomponents of the iTUG task, i.e. the gait, turning, sit-to-stand, turn-to-sit. The details of algorithms used to extract iTUG parameters can be found in [17]. The list of extracted parameters is shown in Table 1 and Appendix.

\section{iSway}

In this instrumented version of a postural sway test, participants were instructed to stand comfortably with their arms folded across their chest. Participants were subsequently instructed to look straight ahead focusing on a fixed target standing still for thirty seconds. Placing a wooden wedge between the patients' feet ensured consistent foot positioning. Forty-seven parameters were calculated using the signals from the sensor placed on the sternum. The parameters of the iSway are grouped under three categories: jerk, time domain and frequency domain parameters. The details of algorithms used to extract iSway parameters can be found in [10]. The list of extracted parameters is shown in Table 2 and Appendix.

\section{Data analysis}

To evaluate within and between sessions reliability of the parameters from the iTUG and iSway tests the Intra-Class Correlation coefficient (ICC) was used. In particular, ICC $(1,1)$ was used for within-session reliability, since the inertial unit, the subjects and the assessor remained the same, whereas $\operatorname{ICC}(2,1)$ was used for

Table 1 Test-retest reliability, mean, Standard Deviation (SD), of parameters with excellent reliability (ICC $(2,1) \geq 0.8)$ from iTUG

\begin{tabular}{|c|c|c|c|c|}
\hline \multirow[t]{2}{*}{ Parameters } & \multirow[t]{2}{*}{ Mean \pm SD } & \multirow{2}{*}{$\begin{array}{l}\text { Test1 } \\
\text { ICC }(1,1)\end{array}$} & \multirow{2}{*}{$\begin{array}{l}\text { Test2 } \\
\text { ICC }(1,1)\end{array}$} & \multirow{2}{*}{$\begin{array}{l}\text { B/W } \\
\text { ICC }(2,1)\end{array}$} \\
\hline & & & & \\
\hline Total Duration (s) & $29.67 \pm 14.31$ & 0.63 & 0.98 & 0.80 \\
\hline Stride Length (m) & $1.24 \pm 0.15$ & 0.78 & 0.98 & 0.81 \\
\hline Stride Velocity (\%statures) & $62.21 \pm 15.10$ & 0.86 & 0.98 & 0.82 \\
\hline RoM Shank (deg.) & $68.66 \pm 10.10$ & 0.93 & 0.98 & 0.80 \\
\hline RoM Knee (deg.) & $53.78 \pm 4.81$ & 0.97 & 0.93 & 0.81 \\
\hline Peak Swing Velocity (deg./s) & $321.93 \pm 59.27$ & 0.94 & 0.98 & 0.84 \\
\hline Cadence (steps/min) & $99.63 \pm 14.23$ & 0.84 & 0.97 & 0.83 \\
\hline RoM Trunk horizontal (deg.) & $6.29 \pm 2.90$ & 0.96 & 0.92 & 0.80 \\
\hline Peak Frontal Trunk Velocity (deg./.s) & $35.85 \pm 13.80$ & 0.92 & 0.97 & 0.80 \\
\hline
\end{tabular}


Table 2 Test-retest reliability, mean, Standard Deviation (SD), of parameters with excellent reliability $($ ICC $(2,1) \geq 0.8)$ from iSway

\begin{tabular}{|c|c|c|c|c|}
\hline \multirow[t]{2}{*}{ Parameters } & \multirow[t]{2}{*}{ Mean \pm SD } & Test1 & Test2 & $\mathrm{B} / \mathrm{W}$ \\
\hline & & $\operatorname{ICC}(1,1)$ & ICC $(1,1)$ & $\operatorname{ICC}(2,1)$ \\
\hline \multicolumn{5}{|l|}{ Time Domain Parameters } \\
\hline RMS sway (ms-2) & $0.196 \pm 0.17$ & 0.84 & 0.88 & 0.83 \\
\hline Mean distance (mm) & $0.154 \pm 0.13$ & 0.84 & 0.9 & 0.83 \\
\hline Mean distance ML (mm) & $0.07 \pm 0.08$ & 0.85 & 0.91 & 0.86 \\
\hline Path length AP (mm) & $12.17 \pm 12.86$ & 0.89 & 0.90 & 0.87 \\
\hline Total sway area (m2/s5) & $0.08 \pm 0.19$ & 0.78 & 0.88 & 0.80 \\
\hline RMS sway ML (ms-2) & $0.10 \pm 0.13$ & 0.86 & 0.87 & 0.86 \\
\hline Path length (mm) & $19.86 \pm 28.36$ & 0.89 & 0.93 & 0.91 \\
\hline Path length ML (mm) & $12.85 \pm 23.69$ & 0.89 & 0.94 & 0.92 \\
\hline Range of acceleration (ms-2) & $1.42 \pm 1.62$ & 0.84 & 0.84 & 0.83 \\
\hline Range of acceleration ML (ms-2) & $0.94 \pm 1.48$ & 0.84 & 0.81 & 0.83 \\
\hline \multicolumn{5}{|l|}{ Jerk Parameters } \\
\hline Jerk AP $\left(\mathrm{m}^{2} / \mathrm{s}^{5}\right)$ & $0.16 \pm 0.50$ & 0.93 & 0.84 & 0.85 \\
\hline Jerk $\left(m^{2} / s^{5}\right)$ & $10.08 \pm 38.12$ & 0.91 & 0.86 & 0.89 \\
\hline Jerk $M L\left(m^{2} / s^{5}\right)$ & $0.52 \pm 2.07$ & 0.88 & 0.86 & 0.88 \\
\hline \multicolumn{5}{|l|}{ Frequency Domain Parameters } \\
\hline Mean frequency AP $(\mathrm{Hz})$ & $0.6 \pm 0.32$ & 0.89 & 0.81 & 0.83 \\
\hline Mean frequency $(\mathrm{Hz})$ & $0.62 \pm 0.30$ & 0.88 & 0.86 & 0.84 \\
\hline Total power ((ms-2) $\left.2^{*} \mathrm{~Hz}-1\right)$ & $13.85 \pm 30.11$ & 0.75 & 0.9 & 0.80 \\
\hline Total power ML ((ms-2)2*Hz-1) & $17.90 \pm 51.22$ & 0.86 & 0.88 & 0.86 \\
\hline High frequency power AP $\left((m s-2) 2^{*} \mathrm{~Hz}-1\right)$ & $18.76 \pm 24.10$ & 0.93 & 0.79 & 0.80 \\
\hline Low frequency power AP ((ms-2) $\left.2^{*} \mathrm{~Hz}-1\right)$ & $367.23 \pm 78.8$ & 0.93 & 0.79 & 0.80 \\
\hline
\end{tabular}

between sessions reliability. The following validated thresholds for ICC values were used: ICC $\geq 0.8$ excellent, $0.6<$ ICC $<0.8$ good, $0.4 \leq \mathrm{ICC} \leq 0.6$ moderate and ICC $<0.4$ poor reliability. The ability of parameters to classify falls status based on falls history of a participant was also assessed using self-reported questionnaire of falls in the last 12 months.

Normality of the data was assessed using the Kolmogorov-Smirnov test. A widely used discriminant classifier model with stepwise function was performed to assess the degree to which different parameters were able to identify fall status $[16,18-20]$. The discriminant function was based on Mahanolobis distance of each variable and was based on leave-one-out validation method. Statistical analysis was carried out in SPSS v22 and MATLAB (Math works, R2014a), with a level of significance set to $p=0.05$.

\section{Results}

All patients included in the study were able to complete both the iTUG and the iSway protocols. All experimental sessions lasted less than fifteen minutes and both operator and participant did not encounter any difficulties.
Results from both tests, ease of use of the system and falls classification are reported below:

\section{iTUG}

In total, 32 parameters were extracted from the iTUG test. Most parameters demonstrated good reliability (Table 1 and Appendix), with mean \pm Standard Deviation (SD) ICC values were :0.81 \pm 0.17 for within and $0.69 \pm 0.15$ for between sessions, respectively. Out of all the parameters, nine showed excellent, sixteen good, five moderate and two poor reliability.

By looking individually at the task components, it was found that the mean ICC (between sessions) values of $0.76 \pm 0.05$ for the gait component, $0.62 \pm 0.10$ for the turning component $0.31 \pm 0.08$ for the sit-to-stand component, and $0.67 \pm 0.07$ for the turn-to-sit component, respectively.

\section{iSway}

Forty-seven parameters were extracted from the iSway test (Table 2 and Appendix). The mean ICC values of iSway parameters for within and between sessions were $0.76 \pm 0.13$ and $0.71 \pm 0.14$, respectively. More specifically, twenty-seven showed excellent, fifteen good, three 
moderate and two poor reliability values. Mean ICC (between sessions) values of the individual category of parameters of iSway were: $0.76 \pm 0.14$ for the time domain parameters, $0.68 \pm 0.13$ for the frequency domain parameters and $0.76 \pm 0.12$ for the jerk component.

\section{Association with falls history}

We explored the added value of parameters from both the tests to classify retrospective falls status of participants in the past 12 months.

In the selection process, we only included parameters which had excellent reliability. Nineteen parameters from iTUG and eighteen parameters from iSway had excellent reliability (shown in Tables 1 and 2). All 37 parameters with excellent reliability were added as an input to the classifier. Interestingly, none of the parameters from iTUG was significant and therefore excluded, only 'Mean distance of the COP $(\mathrm{mm})$ ' from iSway $(p=0.036)$ was selected by the classifier.

Table 3 shows the summary of classification performance of the classifier. The sensitivity of the classifier was $55 \%$, specificity was $100 \%$, overall accuracy was $66.67 \%$ and with an area under the curve of 0.79. Boxplot of Mean distance from COP is shown in Fig. 1.

\section{Sensors' ease of use and timing}

The questionnaires on ease of use were completed by all twenty-seven participants and five operators (operators included were an engineer, physiotherapists, a consultant and a junior doctor) on a scale from to 1 to 4 ( 1 being easy and 4 not possible to use), they all gave a score of 1 .

The average time recorded to set up equipment was five minutes with tests taking on average ten minutes followed by a two-minute download period at the end of the each assessment.

\section{Discussion}

The aim of this small study was to investigate the feasibility of using a commercially available wearable inertial sensor system in a busy clinical environment and to assess the reliability of the gait and balance parameters from the system in order to set up a larger study. A commercial wearable system known as OPAL APDM Inc. with the associated Mobility Lab ${ }^{\text {Tw }}$ software was used to acquire and process the data.

Table 3 Confusion matrix of the classifier

\begin{tabular}{lll}
\hline & Fallers & Non-fallers \\
\hline Fallers & 11 & 9 \\
Non-fallers & 0 & 7 \\
\hline
\end{tabular}

An instrumented version of two widely used tests to assess gait and balance function, the TUG and Sway tests, was used to test the patients' walking and balance ability. The ability of the patients to perform these tests is usually evaluated using score associated with ordinal scales, but the addition of the sensors provides more objective and reliable results $[8,12,21,22]$.

The main contribution of the study was to assess the reliability of extracted parameters robustly and investigate the potential for fall classification. Most of the previous studies have only investigated the within-day reliability of iTUG and iSway parameters $[10,12]$. This study besides the within-day reliability, between-day reliability was also assessed by looking at test-retest data collected one week apart from each other at similar times during the day to ensure the consistency of results of the commercial system.

Several reliable parameters from both the tests and its subcomponents were identified. In particular, the parameters from gait sub-component of the iTUG test were most reliable. 'Peak swing velocity (deg. /s)' of the lower limb and 'Cadence (steps/min)' of gait showed highest between-sessions ICC values of 0.84 and 0.83 respectively. These results are consistent with other studies [12, 17]. The only parameters with $\operatorname{ICC}(2,1)$ greater than 0.80 were: Mean distance mediolateral (ML), Path length anterior-posterior (AP), Root mean square sway ML, Path length and Path length ML whereas the frequency domain parameters were: Total power ML were most reliable, with ICC values greater than 0.85 .

The least reliable part of the iTUG test was the sit-tostand component, which is also been reported in the different populations [23]. A potential cause for poor reliability is the minimum number of constraints imposed on the participants (i.e. use vs. no use of their arms) allowing them to perform the test in a number of different ways. Another reason could be associated with the positioning of the sensors, since even a slight change in their orientation might affect the amplitude of the recorded acceleration and angular velocity signals, and hence the values of the extracted parameters [11].

The parameters 'Path length of sway' the total length of COP trajectory $[\mathrm{mm}]([\mathrm{m} / \mathrm{s} 2])$ in the mediolateral direction (ML) and 'Jerk' $\left(\mathrm{m}^{2} / \mathrm{s}^{5}\right)$ the sway jerkiness showed highest reliability values. These results are similar to findings from Mancini et al. [7] on patients with untreated Parkinson's disease. On comparison with iTUG, The parameters calculated for the iSway demonstrated an overall lower reliability, that may be due to the short period taken to complete the test (30 s) [24]. This study was conducted in the view for potential study with large population size and we propose to extend the iSway duration to $60 \mathrm{~s}$. 


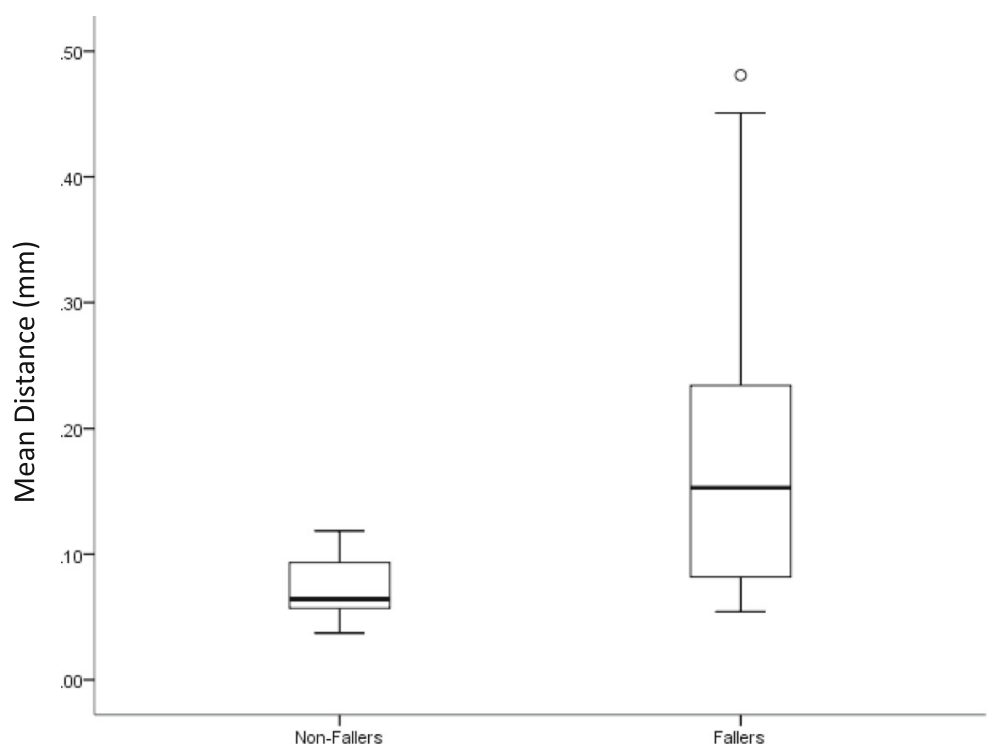

Fig. 1 Boxplot of Mean distance from the COP of fallers and non-fallers

It is unsurprising to see that overall within-session reliability ICC $(1,1)$ of Test 2 is higher than Test 1 . This is due to the participants becoming familiar with the test' administered and were able to perform it with more consistency.

In addition to reliability, the study assessed the ability of reliable parameters to classify fallers. The parameter 'Mean distance from the centre of COP trajectory ( $\mathrm{mm}$ )' from iSway had a significant ability to classify falls status of the participant. Sensitivity and accuracy of the classifier is moderate and this may be due to insufficient information to robustly classify fallers. Although many of the iTUG parameters had excellent reliability they did not have statistical significance to be included in the classification model. Some of the iTUG parameters had a $p$-value slightly over 0.05 set for the study and were excluded by the classifier, this may be due to a lower number of participants in the study. With the larger sample size, it is highly likely that a number of iTUG parameters will be included by the classifier which in turn will increase accuracy and sensitivity of the classification. This is only an initial feasibility study. So only generic falls episodes have been recorded. Falls episodes have other contributors such as environmental, medication use etc. in addition to the vestibular cause. Future studies will accurately record comprehensive falls episodes prospectively.

Despite the limitations, this study showed that it is feasible to use the inertial sensors for the assessment of patients with vestibular disorders in a busy clinical environment. The results of the study show that the system is easy, quick to setup and use in the busy clinical environment. Participants and operators did not find any issues using the system. Most importantly, several parameters from the system have good to excellent reliability. In addition, this study showed that parameters from Mobility lab ${ }^{\mathrm{Tm}}$ system can retrospectively classify falls in these patients in almost $70 \%$ of the case. This commercially available system has the potential for a use in a clinical environment and beyond with its capabilities of portability.

\section{Conclusions}

Use of inertial sensors allows for an objective assessment of gait, stability, balance and functional abilities of an individual both in their natural and the clinical environment. The wearable sensors are non-intrusive, reliable, user-friendly and provide valuable information on the functional ability of vestibular patients. This study used one of the commercially available sensor system known as Mobility Lab $^{\mathrm{Tm}}$ and investigated its feasibility, test-retest reliability in a neurotology clinics. The results show that it is easy to setup and feasible to use in clinical settings. The parameters of the system showed good to excellent reliability and a potential role in falls prediction. These preliminary results show an excellent potential towards more personalised and targeted interventions with an objective evaluation of their efficiency. Future longitudinal, prospective studies will focus on testing the validity and sensitivity of the chosen parameters to actually discriminate amongst individuals from within the same patient group and their correlation to the risk of falls. 


\section{Appendix}

Table 4 Test-retest reliability, mean, Standard Deviation (SD), of all other parameters from iTUG

\begin{tabular}{|c|c|c|c|c|c|c|c|c|}
\hline \multirow[t]{4}{*}{ Parameters } & \multicolumn{4}{|l|}{ Control group } & \multicolumn{4}{|l|}{ Patients group } \\
\hline & \multirow[t]{3}{*}{ Mean \pm SD } & \multirow{3}{*}{$\begin{array}{l}\text { Test1 } \\
\text { ICC } \\
(1,1)\end{array}$} & \multirow{3}{*}{$\begin{array}{l}\text { Test2 } \\
\text { ICC } \\
(1,1)\end{array}$} & \multirow{3}{*}{$\begin{array}{l}\text { B/W } \\
\text { ICC } \\
(2,1)\end{array}$} & \multirow[t]{3}{*}{ Mean \pm SD } & \multirow{3}{*}{$\begin{array}{l}\text { Test1 } \\
\text { ICC }(1,1)\end{array}$} & \multirow{3}{*}{$\begin{array}{l}\text { Test2 } \\
\text { ICC } \\
(1,1)\end{array}$} & \multirow{3}{*}{$\begin{array}{l}\text { B/W } \\
\text { ICC } \\
(2,1)\end{array}$} \\
\hline & & & & & & & & \\
\hline & & & & & & & & \\
\hline Stride velocity (m/s) & $1.35 \pm 0.13$ & 0.96 & 0.95 & 0.88 & $1.04 \pm 0.19$ & 0.77 & 0.98 & 0.77 \\
\hline Double support (\%) & $20.66 \pm 3.16$ & 0.89 & 0.87 & 0.68 & $27.09 \pm 7.70$ & 0.71 & 0.96 & 0.70 \\
\hline Swing (\%) & $39.67 \pm 1.58$ & 0.89 & 0.87 & 0.68 & $36.45 \pm 3.85$ & 0.71 & 0.96 & 0.70 \\
\hline Stance (\%) & $60.33 \pm 1.58$ & 0.89 & 0.87 & 0.68 & $63.55 \pm 3.85$ & 0.71 & 0.96 & 0.70 \\
\hline RoM trunk frontal (deg.) & $17.11 \pm 8.34$ & 0.99 & 0.99 & 0.62 & $9.58 \pm 3.40$ & 0.92 & 0.87 & 0.71 \\
\hline Gait cycle time(s) & $1.12 \pm 0.10$ & 0.98 & 0.96 & 0.90 & $1.24 \pm 0.23$ & 0.66 & 0.96 & 0.78 \\
\hline Peak arm swing velocity (deg./s) & $167.91 \pm 42.7$ & 0.89 & 0.84 & 0.79 & $148.79 \pm 55.55$ & 0.8 & 0.92 & 0.71 \\
\hline RoM arm (deg.) & $21.23 \pm 8.84$ & 0.89 & 0.92 & 0.68 & $18.85 \pm 9.06$ & 0.9 & 0.92 & 0.78 \\
\hline RoM trunk sagittal (deg.) & $6.27 \pm 2.24$ & 0.92 & 0.97 & 0.40 & $5.00 \pm 1.64$ & 0.89 & 0.92 & 0.75 \\
\hline Peak horizontal trunk velocity (deg./s) & $20.34 \pm 7.20$ & 0.96 & 0.93 & 0.83 & $25.89 \pm 11.34$ & 0.98 & 0.9 & 0.71 \\
\hline Peak sagittal trunk velocity (deg./s) & $30.47 \pm 5.94$ & 0.89 & 0.90 & 0.67 & $26.95 \pm 12.21$ & 0.91 & 0.97 & 0.67 \\
\hline Peak frontal trunk velocity (deg./.s) & $45.02 \pm 14.15$ & 0.98 & 0.97 & 0.75 & $35.85 \pm 13.80$ & 0.92 & 0.97 & 0.80 \\
\hline \multicolumn{9}{|l|}{ Turning } \\
\hline Turn duration (s) & $1.93 \pm 0.36$ & 0.60 & 0.71 & 0.41 & $3.48 \pm 1.85$ & 0.58 & 0.92 & 0.70 \\
\hline Turn number of steps & $3.78 \pm 0.80$ & 0.38 & 0.24 & 0.21 & $5.36 \pm 1.91$ & 0.48 & 0.75 & 0.58 \\
\hline Turn peak velocity(deg./s) & $171.69 \pm 26.3$ & 0.45 & 0.70 & 0.37 & $114.12 \pm 36.46$ & 0.81 & 0.91 & 0.75 \\
\hline Turn step time (s) & $0.63 \pm 0.09$ & 0.61 & 0.36 & 0.53 & $0.79 \pm 0.28$ & 0.59 & 0.72 & 0.55 \\
\hline Turn step time before turn (s) & $0.57 \pm 0.05$ & 0.88 & 0.82 & 0.81 & $0.66 \pm 0.18$ & 0.46 & 0.84 & 0.52 \\
\hline \multicolumn{9}{|l|}{ Sit to stand } \\
\hline Duration (s) & $2.30 \pm 0.72$ & -0.03 & 0.26 & 0.04 & $2.72 \pm 1.62$ & 0.17 & 0.27 & 0.26 \\
\hline Peak velocity (deg./s) & $97.45 \pm 24.13$ & 0.52 & 0.72 & 0.54 & $91.29 \pm 38.21$ & 0.44 & 0.41 & 0.26 \\
\hline RoM trunk (deg.) & $38.01 \pm 7.81$ & 0.51 & 0.72 & 0.61 & $32.61 \pm 9.52$ & 0.55 & 0.81 & 0.40 \\
\hline \multicolumn{9}{|l|}{ Turn to sit } \\
\hline Turn to sit Duration (s) & $3.89 \pm 0.72$ & 0.59 & 0.63 & 0.48 & $5.21 \pm 2.13$ & 0.6 & 0.88 & 0.72 \\
\hline Turn to sit RoM trunk (deg.) & $24.97 \pm 8.55$ & 0.90 & 0.82 & 0.58 & $24.60 \pm 7.86$ & 0.71 & 0.77 & 0.59 \\
\hline
\end{tabular}


Table 5 Test-retest reliability, mean, Standard Deviation (SD), of all other parameters from iSway

\begin{tabular}{|c|c|c|c|c|c|c|c|c|}
\hline \multirow[t]{4}{*}{ Parameters } & \multicolumn{4}{|l|}{ Control group } & \multicolumn{4}{|l|}{ Patients group } \\
\hline & \multirow[t]{3}{*}{ Mean $\pm S D$} & Test1 & Test2 & $\mathrm{B} / \mathrm{W}$ & Mean \pm SD & Test1 & Test2 & B/W \\
\hline & & ICC & ICC & ICC & & $\operatorname{ICC}(1,1)$ & ICC & ICC $(2,1)$ \\
\hline & & $(1,1)$ & $(1,1)$ & $(2,1)$ & & & $(1,1)$ & \\
\hline \multicolumn{9}{|l|}{ Time domain parameters } \\
\hline RMS sway AP (ms-2) & $0.066 \pm 0.032$ & 0.63 & 0.64 & 0.55 & $0.157 \pm 0.13$ & 0.82 & 0.87 & 0.77 \\
\hline Mean distance AP (mm) & $0.053 \pm 0.026$ & 0.64 & 0.67 & 0.56 & $0.121 \pm 0.09$ & 0.84 & 0.86 & 0.79 \\
\hline @95circle sway area (m2/s4) & $0.060 \pm 0.071$ & 0.56 & 0.25 & 0.27 & $0.70 \pm 1.36$ & 0.70 & 0.86 & 0.73 \\
\hline @95ellipse sway area (m2/s4) & $0.033 \pm 0.039$ & 0.53 & 0.24 & 0.28 & $0.48 \pm 0.91$ & 0.65 & 0.80 & 0.70 \\
\hline Mean velocity AP (ms-1) & $0.131 \pm 0.091$ & 0.35 & 0.29 & 0.30 & $0.24 \pm 0.23$ & 0.46 & 0.67 & 0.41 \\
\hline Mean velocity (ms-1) & $0.141 \pm 0.092$ & 0.42 & 0.31 & 0.34 & $0.28 \pm 0.25$ & 0.50 & 0.79 & 0.48 \\
\hline Mean velocity ML(ms-1) & $0.035 \pm 0.023$ & 0.20 & 0.39 & 0.26 & $0.10 \pm 0.10$ & 0.63 & 0.66 & 0.55 \\
\hline Range of acceleration AP (ms-2) & $0.348 \pm 0.218$ & 0.43 & 0.07 & 0.28 & $0.95 \pm 0.81$ & 0.78 & 0.82 & 0.73 \\
\hline \multicolumn{9}{|l|}{ Jerk Parameters } \\
\hline Normalized jerk APAD & $1.143 \pm 0.293$ & 0.38 & 0.43 & 0.42 & $1.34 \pm 0.54$ & 0.80 & 0.71 & 0.73 \\
\hline Normalized jerk AD & $4.89 \pm 1.096$ & 0.43 & 0.45 & 0.45 & $5.34 \pm 1.84$ & 0.78 & 0.78 & 0.74 \\
\hline Normalized jerk MLAD & $2.15 \pm 0.663$ & 0.54 & 0.63 & 0.49 & $1.82 \pm 0.53$ & 0.62 & 0.61 & 0.56 \\
\hline \multicolumn{9}{|l|}{ Frequency Domain Parameters } \\
\hline Mean frequency ML (Hz) & $1.142 \pm 0.416$ & 0.70 & 0.76 & 0.57 & $1.03 \pm 0.39$ & 0.82 & 0.84 & 0.78 \\
\hline Centroidal frequency AP (Hz) & $0.595 \pm 0.242$ & 0.37 & 0.59 & 0.49 & $0.7 \pm 0.39$ & 0.90 & 0.81 & 0.77 \\
\hline Centroidal frequency $\mathrm{ML}(\mathrm{Hz})$ & $1.163 \pm 0.363$ & 0.72 & 0.8 & 0.51 & $1.08 \pm 0.35$ & 0.71 & 0.70 & 0.67 \\
\hline Frequency dispersion AP AD & $0.801 \pm 0.054$ & 0.17 & 0.07 & 0.19 & $0.78 \pm 0.07$ & 0.61 & 0.61 & 0.55 \\
\hline Frequency dispersion AD & $0.762 \pm 0.048$ & 0.35 & 0.13 & 0.07 & $0.74 \pm 0.06$ & 0.37 & 0.30 & 0.26 \\
\hline Frequency dispersion ML AD & $0.705 \pm 0.095$ & 0.50 & 0.57 & 0.39 & $0.68 \pm 0.10$ & 0.65 & 0.75 & 0.65 \\
\hline @95frequencyAP (Hz) & $1.444 \pm 0.612$ & 0.39 & 0.76 & 0.54 & $1.60 \pm 0.76$ & 0.88 & 0.8 & 0.68 \\
\hline @95frequency (Hz) & $1.856 \pm .557$ & 0.36 & 0.72 & 0.52 & $1.99 \pm 0.62$ & 0.73 & 0.67 & 0.60 \\
\hline @95frequencyML (Hz) & $2.601 \pm 0.523$ & 0.70 & 0.80 & 0.52 & $2.39 \pm 0.56$ & 0.64 & 0.60 & 0.55 \\
\hline Total power AP ((ms-2)2*Hz-1) & $1.792 \pm 1.950$ & 0.55 & 0.61 & 0.38 & $16.03 \pm 26.80$ & 0.73 & 0.55 & 0.62 \\
\hline Median frequency AP $(\mathrm{Hz})$ & $0.348 \pm 0.162$ & 0.16 & 0.01 & 0.22 & $0.44 \pm 0.34$ & 0.6 & 0.73 & 0.68 \\
\hline Median frequency $(\mathrm{Hz})$ & $0.425 \pm 0.105$ & 0.32 & 0.12 & 0.25 & $0.49 \pm 0.16$ & 0.63 & 0.45 & 0.51 \\
\hline Median frequency ML $(\mathrm{Hz})$ & $0.719 \pm 0.419$ & 0.54 & 0.59 & 0.39 & $0.74 \pm 0.43$ & 0.63 & 0.75 & 0.66 \\
\hline High frequency power $\mathrm{ML}\left((\mathrm{ms}-2) 2^{*} \mathrm{~Hz}-1\right)$ & $54.059 \pm 21.90$ & 0.68 & 0.78 & 0.60 & $38.39 \pm 26.50$ & 0.93 & 0.85 & 0.74 \\
\hline Low frequency power ML ((ms-2) $\left.2^{*} \mathrm{~Hz}-1\right)$ & $251.92 \pm 71.5$ & 0.67 & 0.78 & 0.59 & $303.49 \pm 86.9$ & 0.93 & 0.85 & 0.74 \\
\hline
\end{tabular}




\section{Abbreviations}

BPPV: Benign paroxysmal positional vertigo; CDP: Computerised dynamic posturography; COP: Centre of pressure; ENG: Electronystagmography; ICC: Intraclass correlation coefficient; Isway: Instrumented sway; iTUG: Instrumented Timed Up and Go; MIMUs: Magnetic inertial measurement units; ML: Mediolateral; SD: Standard deviation; VNG: Videonystagmography.

\section{Acknowledgements}

We are especially grateful to all the participants of the study.

\section{Funding}

This study was funded by the Sheffield Hospitals Charity as part of their "INSIGNEO Bursary for Clinical Translation" scheme and from the "EPSRC Frontier Engineering Awards", Grant Reference No. EP/K03877X/1.

\section{Availability of data and materials}

The data used for this study is available through Figshare https://

figshare.com/articles/FigSharedataOPAL_paper_xlsx/4276376.

\section{Authors' contributions}

SSP: helped to design the study, acquisition of data, analysis and interpretation of data and prepared the manuscript, JR: conceived and designed the study, helped draft the manuscript, ALB: helped data collection and draft the manuscript, CM: conceived and designed the study, helped to analyse and interpret the data and draft the manuscript. All authors read and approved the manuscript.

\section{Competing interests}

The authors declare that they have no competing interests.

\section{Consent for publication}

Not applicable.

\section{Ethics approval and consent to participate}

The study was approved by the National Research Ethics committee West Midlands-Edgbaston, reference number 14/WM/0146. An informed consent was obtained from all the participants of the study.

\section{Author details}

${ }^{1}$ INSIGNEO Institute for in silico Medicine, University of Sheffield, Sheffield, UK. ${ }^{2}$ School of Health Sciences, University of Salford, Manchester, UK. ${ }^{3}$ Ear, Nose and Throat Surgery Department, Sheffield Teaching Hospitals, Sheffield, UK. ${ }^{4}$ Department of Mechanical Engineering, University of Sheffield, Sheffield, UK. ${ }^{5}$ University of Salford, 111, Crescent House, England M5 4WT, UK.

Received: 6 June 2016 Accepted: 8 January 2017

Published online: 02 February 2017

\section{References}

1. Agrawal Y, Carey JP, Della Santina CC, Schubert MC, Minor LB. Diabetes, vestibular dysfunction, and falls: analyses from the National Health and Nutrition Examination Survey. Otol Neurotol. 2010;31(9):1445-50.

2. Axer $H$, Axer $M$, Sauer $H$, Witte $O W$, Hagemann $G$. Falls and gait disorders in geriatric neurology. Clin Neurol Neurosurg. 2010;112(4):265-74.

3. Finlayson ML, Peterson EW. Falls, aging, and disability. Phys Med Rehabi Clin N Am. 2010;21(2):357-73.

4. Pothula VB, Chew F, Lesser TH, Sharma AK. Falls and vestibular impairment Clin Otolaryngol Allied Sci. 2004:29(2):179-82.

5. Scuffham P, Chaplin S, Legood R. Incidence and costs of unintentional falls in older people in the United Kingdom. J Epidemiol Community Health. 2003;57(9):740-4

6. Wuyts FL, Furman J, Vanspauwen R, Van de Heyning P. Vestibular function testing. Curr Opin Neurol. 2007;20(1):19-24.

7. Cohen HS, Cox C, Springer G, Hoffman HJ, Young MA, Margolick JB, Plankey MW. Prevalence of Abnormalities in Vestibular Function and Balance among HIV-Seropositive and HIV-Seronegative Women and Men. PLoS One. 2012; 7(5):e38419.

8. Greene BR, O'Donovan A, Romero-Ortuno R, Cogan L, Scanaill CN, Kenny RA. Quantitative falls risk assessment using the timed up and go test. IEEE Trans Biomed Eng. 2010;57(12):2918-26.
9. Higashi Y, Yamakoshi K, Fujimoto T, Sekine M, Tamura T. Quantitative evaluation of movement using the timed up-and-go test. IEEE Eng Med Bio Mag. 2008;27(4):38-46

10. Mancini M, Salarian A, Carlson-Kuhta P, Zampieri C, King L, Chiari L, Horak FB. ISway: a sensitive, valid and reliable measure of postural control. Neuroeng Rehabil. 2012;9:59.

11. Salarian A, Russmann H, Vingerhoets FJ, Dehollain C, Blanc Y, Burkhard PR, Aminian K. Gait assessment in Parkinson's disease: toward an ambulatory system for long-term monitoring. IEEE Trans Biomed Eng. 2004;51(8):1434-43.

12. Zampieri C, Salarian A, Carlson-Kuhta P, Aminian K, Nutt JG, Horak FB. The instrumented timed up and go test: potential outcome measure for disease modifying therapies in Parkinson's disease. J Neurol Neurosurg Psychiatry. 2010:81(2):171-6.

13. Cohen HS, Mulavara AP, Peters BT, Sangi-Haghpeykar H, Bloomberg JJ. Standing balance tests for screening people with vestibular impairments. Laryngoscope. 2014;124(2):545-50.

14. Mancini M, King L, Salarian A, Holmstrom L, McNames J, Horak FB. Mobility Lab to Assess Balance and Gait with Synchronized Body-worn Sensors. J Bioeng Biomed Sci. 2011;Suppl 1:007.

15. Podsiadlo D, Richardson S. The timed "Up \& Go": a test of basic functional mobility for frail elderly persons. J Am Geriatr Soc. 1991;39(2):142-8.

16. Shumway-Cook A, Brauer S, Woollacott M. Predicting the probability for falls in community-dwelling older adults using the Timed Up \& Go Test. Phys Ther. 2000:80(9):896-903.

17. Salarian A, Horak FB, Zampieri C, Carlson-Kuhta P, Nutt JG, Aminian K. iTUG, a Sensitive and Reliable Measure of Mobility. IEEE Trans Neural Syst Rehabil Eng. 2010;18(3):303-10.

18. Blake AJ, Morgan K, Bendall MJ, Dallosso H, Ebrahim SBJ, Arie THD, Fentem $\mathrm{PH}$, Bassey EJ. Falls by elderly people at home: Prevalence and associated factors. Age Ageing. 1988;17(6):365-72.

19. Maeda N, Urabe Y, Murakami M, Itotani K, Kato J. Discriminant analysis for predictor of falls in stroke patients by using the Berg Balance Scale. Singapore Med J. 2015:56(5):280-3.

20. Lord SR, Ward JA, Williams P, Anstey KJ. Physiological factors associated with falls in older community-dwelling women. J Am Geriatr Soc. 1994; 42(10):1110-7

21. Prieto TE, Myklebust JB, Hoffmann RG, Lovett EG, Myklebust BM. Measures of postural steadiness: differences between healthy young and elderly adults. IEEE Trans Biomed Eng. 1996:43(9):956-66.

22. Mancini M, Horak FB. The relevance of clinical balance assessment tools to differentiate balance deficits. Eur J Phys Rehabil Med. 2010:46(2):239-48.

23. Botolfsen P, Helbostad JL, Moe-Nilssen R, Wall JC. Reliability and concurrent validity of the Expanded Timed Up-and-Go test in older people with impaired mobility. Physiother Res Int. 2008;13(2):94-106.

24. Lafond D, Corriveau H, Hebert R, Prince F. Intrasession reliability of center of pressure measures of postural steadiness in healthy elderly people. Arch Phys Med Rehabil. 2004;85(6):896-901.

\section{Submit your next manuscript to BioMed Central and we will help you at every step:}

- We accept pre-submission inquiries

- Our selector tool helps you to find the most relevant journal

- We provide round the clock customer support

- Convenient online submission

- Thorough peer review

- Inclusion in PubMed and all major indexing services

- Maximum visibility for your research

Submit your manuscript at www.biomedcentral.com/submit
Biomed Central 